\title{
Effect of Merocyanine 540 on Langmuir-Blodgett films and liposomes of zwitterionic, anionic and cationic lipid composition
}

\author{
M. Alay, J. Prat, M.A. Alsina and M.A. Busquets \\ Physical Chemistry Department, Faculty of Pharmacy, Av. Joan XXIII, s/n, \\ 08028 Barcelona, Spain
}

\begin{abstract}
The interaction of the anionic probe Merocyanine 540 (MC540) with mono and bilayers of different lipid composition has been analyzed in order to get insight in the lipid charge influence on probe activity. The Langmuir-Blodgett technique showed that MC540 was able to incorporate predominantly into cationic lipid monolayers composed of Dimyristoyl phosphatidyl choline/ Dimyristoyl trimethyl ammoniumpropane (DMPC/DMPTAP) (75:25, mol:mol). However, at low surface pressures, the probe could also allocate into the interface of the zwitterionic Dimyristoyl phosphatidyl choline (DMPC) and the anionic Dimyristoyl phosphatidyl choline/ Dimyristoyl phosphatidyl glycerol (DMPC/ DMPG) (75:25, mol:mol) thus indicating a low interaction with these lipid compositions. These results were in agreement with the studies performed with Large Unilamelar Vesicles (LUVs) of the same lipid compositions based in the spectroscopic analysis of probe absorption maxima modification upon its incorporation into the lipid bilayers.
\end{abstract}

\section{INTRODUCTION}

Merocyanine 540 is an amphipathic anionic probe that has been shown to be useful to examine lipid head group spacing as well as the surface properties of mono and bilayers $[1,2]$. Probe incorporation into the monolayers depends strongly on the lipid composition [3]. In this work, we use lipid monolayers of different charge Dimyristoyl phosphatidyl choline (DMPC) (zwiterionic), Dimyristoyl phosphatidyl choline/ Dimyristoyl phosphatidyl glycerol (DMPC/ DMPG) (75:25, mol:mol) (anionic) or Dimyristoyl phosphatidyl choline/ Dimyristoyl trimethyl ammonium-propane (DMPC/DMPTAP) (75:25, mol:mol) (cationic) to investigate the influence of neutral, negative and positive charge in the interaction of the dye with model membranes. This work is in agreement with the studies performed with Large unilamelar vesicles (LUVs) of the same composition where various probe to phospholipid molecular ratios were analysed by spectroscopic techniques. The increase in the lipid/dye ratio results in a bathochromic shift concomitant with and hiperchromic effect.

\section{MATERIALS AND METHODS}

\subsection{Chemicals}

Dimyristoyl phosphatidyl choline (DMPC), Dimyristoyl trimethyl ammonium propane (DMPTAP), Dimyristoyl phosphatidyl glycerol (DMPC/DMPG) were from Avanti Lipids and were used without further purification. Merocianyne 540 (MC540) was from Sigma. Chloroform and methanol (pro analysis) were from Merck. Water was double distilled and deionized (Milli-Q system, Millipore Corp.). Tris $10 \mathrm{mM}, \mathrm{pH}$ 7.4 was used throughout the experiment.

\subsection{Compression isotherms (curves area/pressure)}

Lipids from a chloroform/ methanol $(2: 1, \mathrm{v} / \mathrm{v})$ solution $(1 \mathrm{mg} / \mathrm{ml})$ were spread on a buffered subphase Tris $10 \mathrm{mM}$, pH 7.4, containing $3.5 \mathrm{mM}$ merocyanine 540 . Control experiments were done in the same way but without probe into the subphase. After $10 \mathrm{~min}$ allowed for solvent evaporation, 
films were compressed symmetrically up to their collapse pressure using two Teflon moving barriers at a speed of $50 \mathrm{~cm}^{2} / \mathrm{min}$ on a Langmuir Blodgett system (Nima Coventry. UK). Each run was repeated three times and the reproducibility was $0.01 \mathrm{~nm}^{2} /$ molecule. The temperature of the subphase was $21 \pm 0.5^{\circ} \mathrm{C}$.

\subsection{Spectroscopic studies}

Large unilamelar vesicles (LUVs) of Dimyristoyl phosphatidyl choline (DMPC), Dimyristoyl phosphatidyl choline/ Dimyristoyl phosphatidyl glycerol (DMPC/DMPG) (75:25), and Dimyristoyl phosphatidyl choline/Dimyristoyl trimethyl ammonium propane (DMPC/DMPTAP) (75:25), were prepared by the extrusion method [4] (Northern lipids Inc., Canada) by passing the lipid dispersion $(10 \mathrm{mmol} / \mathrm{L}$ Tris, $\mathrm{pH} 7.4) 10$ times trough $25 \mathrm{~mm}$ polycarbonate filters with $0.1 \mu \mathrm{m}$ pore size (Nucleopore Corp., Canada). Liposomes size was $110 \pm 3 \mathrm{~nm}$ with a polydispersity index of $0.01 \pm 0.02 . \mathrm{MC}_{540}$ was incorporated to the lipid bilayer by incubating the probe with the performed LUVs. Absorption spectra were recorded in a Schimadzu UV-2401 PC spectrophotometer, between 400 and $650 \mathrm{~nm}$ with $1-\mathrm{nm}$ steps. Spectra at various lipid/dye molar ratios were recorded at $23 \pm 0.5^{\circ} \mathrm{C}$.

\section{RESULTS AND DISCUSSION}

Figures 1, 2 and 3 show the compression isotherms of the lipids in absence and presence of $\mathrm{MC}_{540}$ in the subphase.
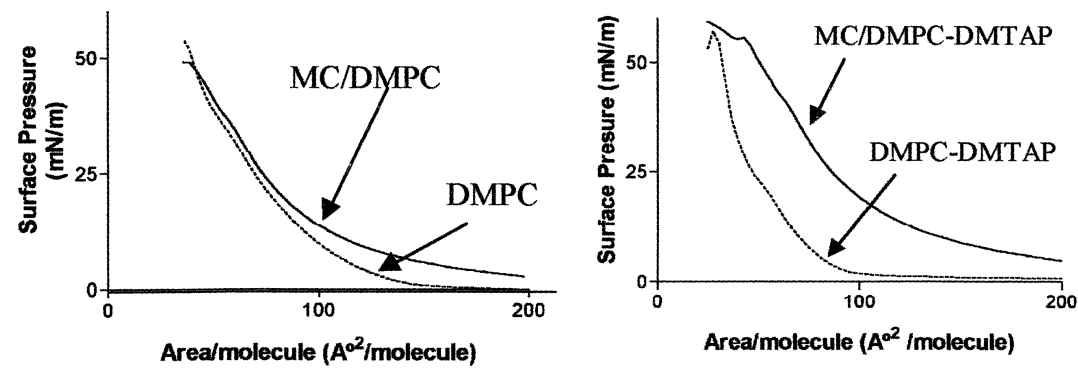

Figure 1: Isotherms of surface pressure vs. mean Figure 2. Isotherms of surface pressure vs. mean molecular area for monolayers of DMPC (zwitterionic molecular area for monolayers of DMPC/DMTAP lipid). Monolayers were formed by spreading the lipids at the interface on a subphase of Tris $10 \mathrm{mM}, \mathrm{pH} 7.4$ alone or containing $\mathrm{MC}_{540}(3.5 \mathrm{mM})$.

For all the lipids tested, we observe an expanding effect of the probe (curves in presence of MC540 are displaced to the left) if compared to the lipid(s) alone, indicating an incorporation of MC into the lipid monolayer. Probe incorporation and thus interaction with lipids is clear at low pressures (high areas), however, for the zwitterionic DMPC and the anionic mixture (DMPC/DMPG) the probe seems to be squizzed out from the monolayer at high surface pressures. At low pressures, the area available for the molecules is big and in the case of DMPG, the electrostatic repulsion is low. When decreasing the area available upon monolayer compression, molecules are closer and the negative charges of MC540 and DMPG result in an electrostatic repulsion. Merocyanine is then expulsed form the surface and solved again into the subphase, while the lipid remains at the 
interface air/water. A similar trend is observed in presence of DMPC although in this case we can not attribute probe expulsion to electrostatic repulsion because of the neutral nature of the lipid.

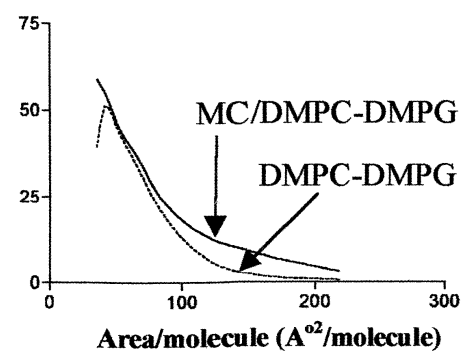

Figure 3. Effect of Merocianyne 540 (3.5mM into Figure 4. Visible absorption spectra of MC540 in subphase) on the anionic mixture DMPC/DMPG suspension of liposomes of DMPC/DMPG $(75: 25$, (75:25, mol:mol).

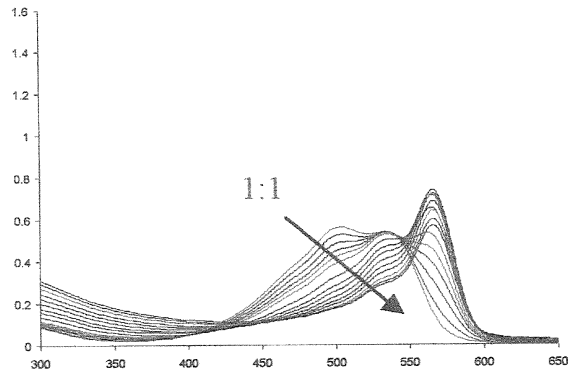
mol:mol).at growing lipide/dye ratio: aqueous solution of MC540 ,1:1, 2:1, 3:1, 5:1, 7:1, 15:1, 20:1, 30:1, 40:1, $50: 1,60: 1,65: 1,70: 1$.

Although merocyanine is able to incorporate into bilayers [3] it seems to have a preferential affinity for the aqueous media. Differences could be due to the formation of J-aggregates depending on the lipid as it has been observed in the case of mixtures of arachidic acid/MC540 [5]. Results obtained in presence of the cationic mixture were expected considering the differences in charge of the lipids and the probe. Pressure in presence of the probe is high before compression and remains much higher than the one of the control during compression. In that case, it looks like a tight interaction between the lipid mixture and the probe as a consequence of the strong attraction between them. These results indicate a clear electrostatic interaction of the probe with the lipids. Data obtained by absorption spectroscopy are in agreement with this conclusion. In aqueous solution MC540, shows two absorption maxima, at $500 \mathrm{~nm}$ (dimer peak) and at $533 \mathrm{~nm}$ (a monomer peak). In the presence of liposomes, the absorption maximun of the two main peaks undergoes a bathochromic shift This suggest a hydrophobic environment of the probe chromophore [6]. As we saw in the isotherms, the presence of anionic charges in liposomes causes an slight electrostatic repulsion, thereby MC540 is expulsed into subphase, this explain that the presence of DMPG in liposomes reduce the quantity of monomer-bound to the membrane, also the absorption maximum of membrane-bound dimer decrease, it may indicate that there is less proportion of dye bound to the membrane.

With the cationic membranes the absoption maximum of membrane-bound dimer increases, it should be caused by a new spatial disposition of MC540 in the membrane that make easy the formation of membrane-bound dimer.

All results indicate a clear electrostatic interaction of the probe with lipids in agreement with results obtained with lipid bilayers analyzed with spectroscopic techniques [3].

These findings are of interest in view to probe application as an external probe in the study o molecules interaction with model membranes.

\section{Acknowledgements}

This work was funded by the grant BQU2000-079-C02-01 from the Ministerio de Ciencia Tecnología (CICYT) (Spain). 


\section{References}

1. M. Langner, S.W. Hui, Biochim. Biophys Acta, 1415, 323 (1999).

2. D.K. Hincha, A.E. Oliver, J.H. Crowe, Biophys. J., 77, 2024 (1999).

3. M. Alay, J. Prat, I. Haro, N. Rojo, M.A. Alsina, M.A. Busquets, Talanta, 60, 269 (2003).

4. New RRC. Preparation of liposomes. IN Liposomes: a Practical Approach, New RRC (ed.) IRL Press: Oxford, 1990, Chapter 2.

5. Y. Hirano, J. Kawata, Y.F. Miura, M. Sugi, T. Ishii, Thin Solid Films, 345, 327 (1998).

6. R. Sabaté, M. Gallardo, J. Estelrich, J. Colloid and Interface Sci., 233, 205 (2002). 